Supplement of Earth Surf. Dynam., 9, 673-685, 2021

https://doi.org/10.5194/esurf-9-673-2021-supplement

(C) Author(s) 2021. CC BY 4.0 License.

(c) (i)

Supplement of

\title{
Precise water level measurements using low-cost GNSS antenna arrays
}

David J. Purnell et al.

Correspondence to: David J. Purnell (david.purnell@ mail.mcgill.ca)

The copyright of individual parts of the supplement might differ from the article licence. 


\section{S1 Combined inversion of SNR data from multiple antennas}

Instead of using the methodology described in Section 2 in the main text for combining data from co-located antennas by averaging $b$-spline scaling factors, we also tried performing inverse analysis using data from all co-located antennas simultaneously. This combined inversion is performed by adapting equation (2) in the main text, such that $h$ is written in terms of the reflector height for a chosen reference antenna and the predetermined vertical separation between each antenna and the reference antenna. The RMSE increases from 1.02 to $1.24 \mathrm{~cm}$ when performing the combined inversion with all four antennas at Trois-Rivières as opposed to averaging $b$-spline scaling factors from different antennas. This result suggests that there is no advantage in performing a combined inversion using data from multiple co-located antennas.

\section{S2 Data processing}

The signals received by the GNSS100L and BN-84U antennas are internally processed and recorded into the National Marine Electronics Association (NMEA) 0183 format, which contains processed GNSS data such as the estimated position of the antenna, number satellites in view and position and strength of signal received from the satellites. This is in contrast to the standard receiver independent exchange (RINEX) format, standard for geodetic receivers, which contains information about the carrier phase of received signals that can be processed to obtain precise positioning data. Whilst precise positioning is not possible with the low-cost antennas used herein, the key parameter of interest for reflectometry applications is the SNR data, which is recorded in the NMEA file format.

The azimuth and elevation angle of satellites relative to the antenna is also recorded in the NMEA file format at a resolution of 1 degree. This resolution is not good enough for obtaining reflector height estimates. Instead, we use the Center for Orbit Determination in Europe (COD) orbit solutions that are available online from the Multi-GNSS Experiment and Pilot Project (MGEX) and Crustal Dynamics Data Information System (CDDIS) archive. These orbit solutions give the location of each GNSS satellite every 5 minutes, which can then be interpolated to every second and converted to an azimuth and elevation angle at the antenna. Codes that were used to obtain and process this data are provided along with this article. 

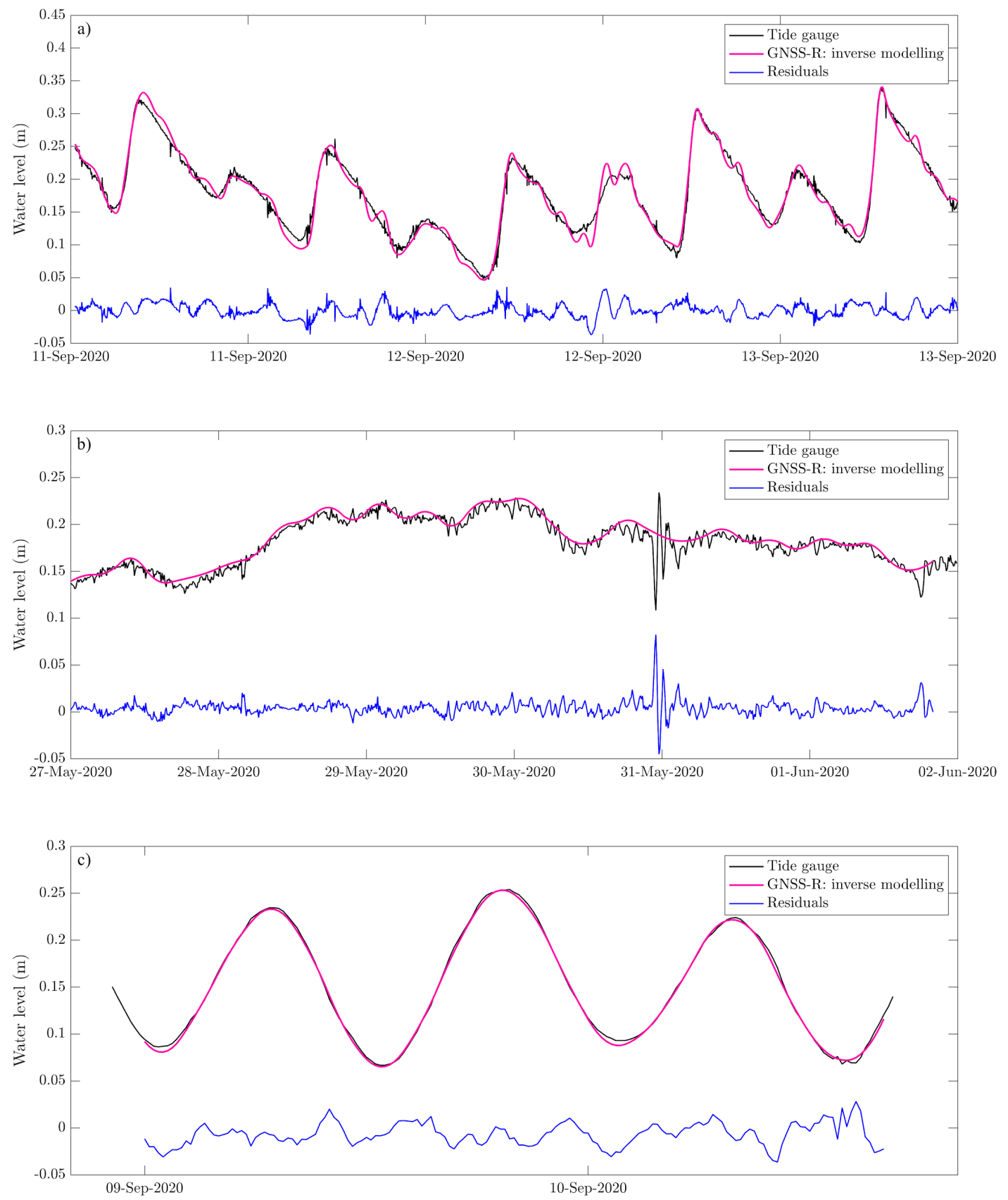

Figure S1: Comparison of GNSS-R and tide gauge measurements with residuals at a) Trois-Rivières, b) Sainte-Anne-de-Bellevue and c) Piermont, as in Figure 4 in the main text but for reduced periods. In each of these figures, the GNSS-R and tide gauge measurements are offset by an arbitrary amount from the residuals. In c), the GNSS-R and tide gauge measurements have been scaled by 0.2 for an easier comparison with the residuals. 